Hydraulic Engineering Repository

Ein Service der Bundesanstalt für Wasserbau

Gebhardt, Michael; Schmitt-Heiderich, Peter

Entwicklung und Simulation von Regelungsalgorithmen für Staustufen an Bundeswasserstraßen

Verfügbar unter / Available at:

https://hdl.handle.net/20.500.11970/104564

Vorgeschlagene Zitierweise / Suggested citation:

Gebhardt, Michael; Schmitt-Heiderich, Peter (2008): Entwicklung und Simulation von

Regelungsalgorithmen für Staustufen an Bundeswasserstraßen. In: Wasserwirtschaft 98 (6). S. 16-18.

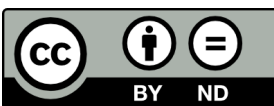


Erstveröffentlichung in Wasserwirtschaft 6 (2008), S. 16-18.

Für eine korrekte Zitierbarkeit ist die Seitennummerierung

der Originalveröffentlichung für jede Seite kenntlich gemacht.

\section{Entwicklung und Simulation von Regelungsalgorithmen für Staustufen an Bundeswasserstraßen}

Die automatische Abfluss- und Stauzielregelung von Stauhaltungen ist aufgrund des nicht-linearen Streckenverhaltens und der Mehrfachnutzung eine anspruchsvolle Regelungsaufgabe. In diesem Beitrag werden die Methoden vorgestellt, die bei der Entwicklungund Simulation von Regelungsalgorithmen für Staustufen an Bundeswasserstraßen verwendet werden. Es wird gezeigt, dass dabei für den Reglerentwurf auf eine Modellierung des Stellorganes Wehr nicht verzichtet werden kann.

\section{Einleitung}

Deutschland verfügt über ein weitmaschiges, wirtschaftlich leistungsfähiges Wasserstraßennetz mit einer Gesamtlänge von etwa $7350 \mathrm{~km}$. Der Wasser- und Schifffahrtsverwaltung des Bundes (WSV) obliegt die Unterhaltung der Bundeswasserstraßen, von denen $24 \%$ kanalisiert, $35 \%$ frei fließend und $41 \%$ staugeregelt sind. Für die Abfluss- und Stauzielregelung betreibt die WSV über 280 Wehranlagen vorrangig zur Verbesserung der Schifffahrtsverhältnisse. In den meisten Fällen befinden sich daneben Kraftwerke von Energieversorgungsunternehmen (EVU), so dass die Stauhaltung einer Mehrfachnutzung mit zum Teil gegensätzlichen Zielsetzungen unterliegt. Ein effizienter bzw. optimierter Betrieb von Staustufen erfordert sie weitestgehend automatisiert zu betreiben.

Im Rahmen der Automatisierung von Staustufen u. a. an Mosel, Saar und Neckar ist es die Aufgabe der Bundesanstalt für Wasserbau (BAW), die Entwicklung und die Parametrisierungder Regelalgorithmen vorzunehmen. Die Randbedingungen dafür ergeben sich aus der Nutzung einer Staustufe durch WSV und EVU: Je nach Wasserdargebot erfolgt der Abfluss über das Wehr und/oder das Kraftwerk. Der Oberwasserstand wird in den meisten Fällen auf einem konstanten Niveau gehalten, je nach Anforderung existieren abfluss-und zeitabhängige Stauziele. Die Stauzieltoleranz beträgt in den meisten Fällen nicht mehr als $\pm 5 \mathrm{~cm}$.

\section{Modellierung von Stauhaltung, Leittechnik und Wehr}

Stand der Technik ist es heute, Stauhaltung, Leittechnik und Wehr in einem mathematischen Modell abzubilden, um den Reglerzu untersuchen und zu parametrisieren, die Auswirkungen auf die Stauhaltung zu beurteilen und Sonderfälle im Staustufenbetrieb gefahrlos testen zu können. Dies erfolgt in der BAW mit der graphisch-interaktiven Entwicklungsumgebung MATLAB/SIMULINK.

Für die Modellierung der Regelstrecke Stauhaltung werden dabei hydrodynamisch-numerische Verfahren (HN-Verfahren; 1D, instationär) mit einer räumlichen Diskretisierung zwischen 50 bis 


\section{Autorenfassung}

Gebhardt, Schmitt-Heiderich: Entwicklung und Simulation von Regelungsalgorithmen für Staustufen an Bundeswasserstraßen, 2008

100 m verwendet. Dem Modell der Leittechnik liegt eine OW/Q-Regelung mit einem PI-Algorithmus (Regelungsanteil) und einer abflussabhängigen Störgrößenaufschaltung (Steuerungsanteil) zugrunde sowie den Filtern zur Glättung der Eingangswerte. Im Modell für das Regelorgan

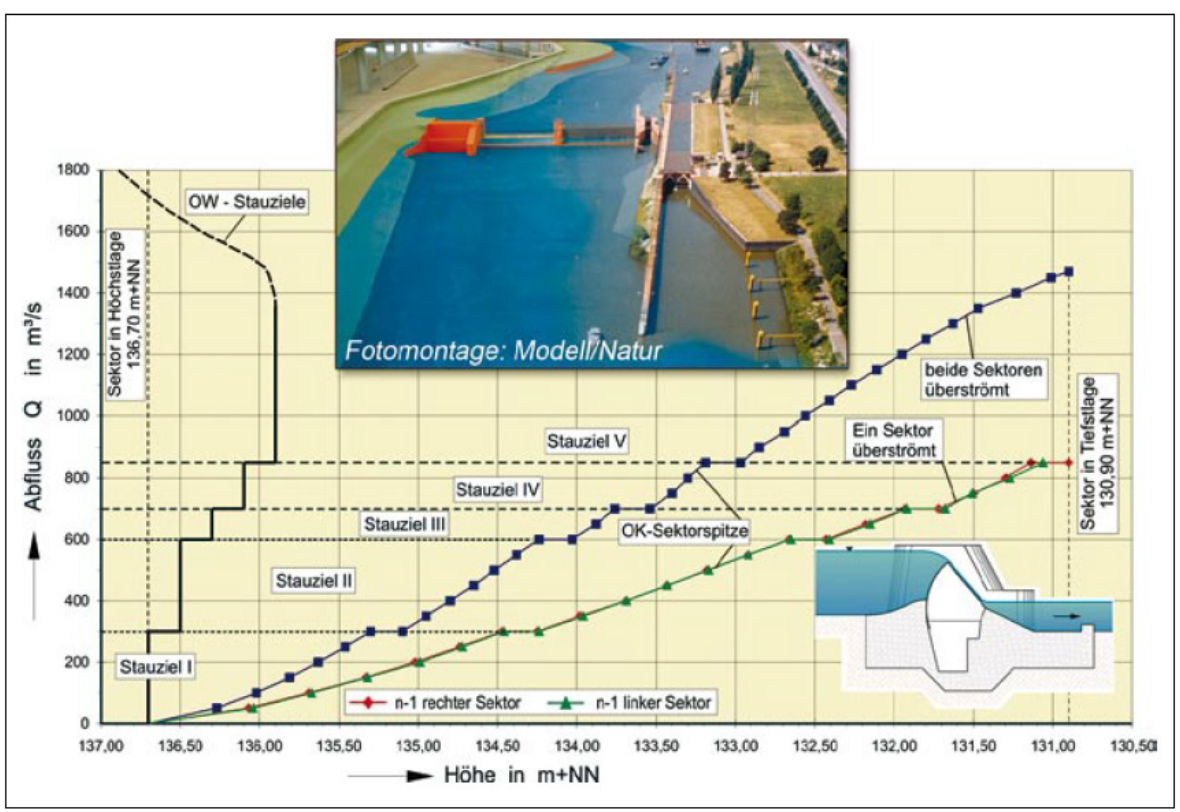

Bild 1: $\quad$ Moselstaustufe Grevenmacher: Wehrkennliniebasierend auf physikalischen Modelluntersuchungen, Fotomontage der Staustufe in Natur und Modell

Gebhardt, Schmitt-Heiderich: Entwicklung und Simulation von Regelungsalgorithmen für Staustufen an Bundeswasserstraßen. Wasserwirtschaft 6 (2008), S. 16-18.

werden neben den Wehrkennlinien, der Wehrfahrplan, die Verstellgeschwindigkeit, minimale und maximale Verstellschrittweiten sowie untere und obere Endlagen berücksichtigt.

Die Automatisierung von Wehranlagen setzt die Kenntnis von Wehrkennlinien voraus, die den spezifischen Zusammenhang zwischen Verschlussstellung, Abfluss, Ober- und Unterwasserstand beschreiben. Mit Hilfe der Wehrkennlinien kann der über den Regler ermittelte Sollabfluss in eine Solllage eines Verschlusses umgerechnet werden und umgekehrt. Bild 1 zeigt beispielhaft die Moselstaustufe Grevenmacher, die aus einem Kraftwerk, einem zweifeldrigen Sektorwehr, einer Kahnschleuse und einer Schifffahrtsschleuse besteht. Die Bestimmung der Wehrkennlinien erfolgte mit einem Labormodell, das nach dem Froudeschen Modellgesetz konzipiert wurde [1]. Die in etwa 80 Versuchsreihen mit unterschiedlichen Randbedingungen ermittelten Wehrkennlinien wurden hier durch Polynome approximiert und in dieser Form in der Speicherprogrammierbaren Steuerung (SPS) implementiert. 


\section{Autorenfassung}

Gebhardt, Schmitt-Heiderich: Entwicklung und Simulation von Regelungsalgorithmen für Staustufen an Bundeswasserstraßen, 2008

\section{Einfluss der Wehrträgheit auf den Regelkreis}

Im Vergleich zu Turbinen weisen Wehrverschlüsse ein träges Verhalten auf, was unter Umständen dazu führt, dass ein neuer Sollwert im nächsten Taktschritt nicht umgesetzt wird. Mit Verstellgeschwindigkeiten von wenigen Zentimetern pro Minute benötigt ein Wehrfeld viele Minuten bis zu einigen Stunden (bauwerksabhängig), um von einer Endlage in die andere zu gelangen. Zur Verringerung des Verschleißes der Antriebsorgane werden die Wehre nur in Mindestverstellschritten bewegt. Auch folgt auf einen Verstellschritt nicht sofort ein weiterer Verstellschritt (Totzeiten). Erfahrungen an der Mosel zeigen, dass die Parametrisierung der Regler in Abhängigkeit von den Kenngrößen der Wehranlage erfolgen muss, da es ansonsten zu einem unerwünschten Aufschaukeln der Stauhaltung kommen kann. Dieses wird nachfolgend mit einem analytischen Ansatz veranschaulicht.

In dem Konzept von Kühne [2]/[3] werden nicht die absoluten Größen betrachtet, sondern lediglich die Änderung der einzelnen Größen gegenüber den stationären Ausgangswerten. Danach bewirkt eine Zuflussänderung f eine Wasserstandsänderung h, die in diesem Ansatz auch der Regelabweichung entspricht. Der PI-Regler mit Eingang h bestimmt die Abflussänderung q. Die Strukturdiagramme für den Ansatz nach Kühne sind in Bild 2 (rot hinterlegt) dargestellt.

Das analytische Modell der Regelstrecke löst die Kontinuitätsgleichung mit einem Speicher, der mit der SpeicheroberflächeA parametrisiert ist. Die Änderungen im Zufluss $f$ und im Abfluss q wirken sich zeitgleich auf die Regelstrecke aus. Diese vereinfachte Beschreibung der Regelstrecke trifft dann näherungsweise zu, wenn am Kraftwerk Turbinen ein bzw. ausgeschaltet werden. Infolge dessen ändert sich der Wasserstand $\mathrm{h}$ im Oberwasser und das Wehr muss reagieren, da der Gesamtabfluss über die Stauanlage gehalten werden muss. Für das Regelorgan Wehr ändert sich der Zufluss schlagartig um die Abflussänderung am Kraftwerk. Die Wehrträgheit wird vereinfachend über ein PT1-Glied beschrieben. Um hochfrequente schiffs- und windinduzierte Wellen aus den Wasserstandsmessungen herauszufiltern, werden die Messwerte mit einem Tiefpass geglättet. Dazu wurden die Strukturdiagramme um die Messwerterfassung und das Regelorgan Wehrerweitert. Daraus ergeben sich die Gleichungen in Bild 2 für die Laplace- Transformierten der Wasserstandsänderung h und Abflussänderung q. Werden die Zeitkonstanten für Wehr und Filter vernachlässigt, erhält man gerade den Ansatz nach Kühne [2].

Die Lösung erfolgt unter der Annahme einer Zuflussänderung f von 100 m³/s. Die 


\section{Autorenfassung}

Gebhardt, Schmitt-Heiderich: Entwicklung und Simulation von Regelungsalgorithmen für Staustufen an Bundeswasserstraßen, 2008

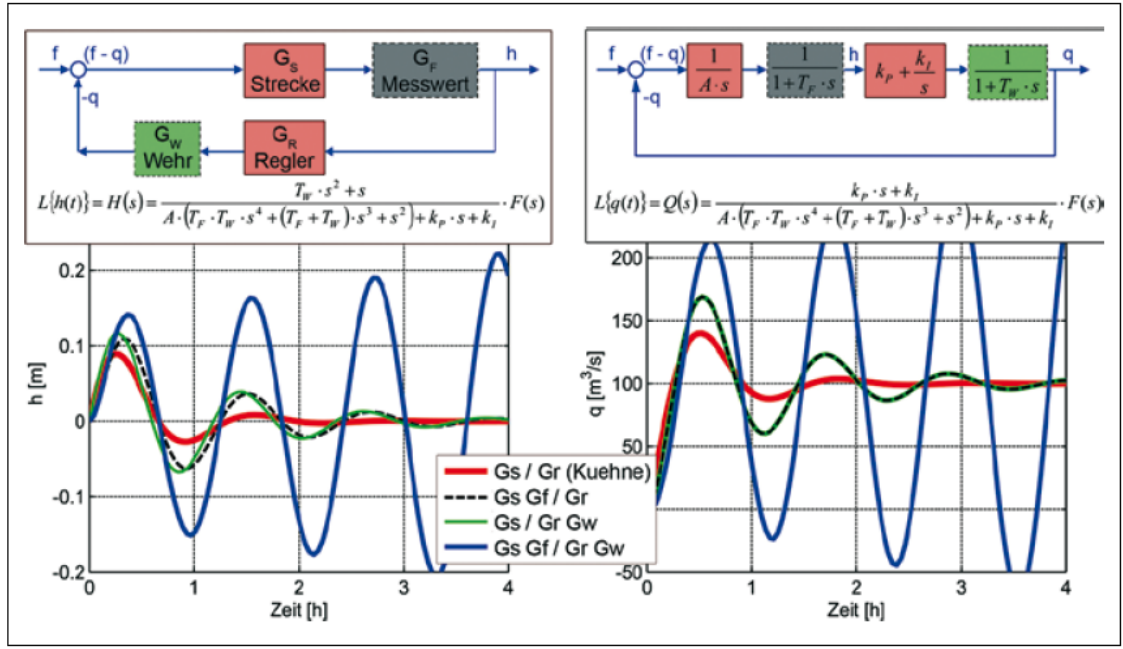

Bild 2: $\quad$ Strukturdiagramme und Berechnungsergebnisse für die Wasserstandsänd erung h und Abflussänderung q bei analytischer Beschreibung der Regelkreise (hier: $\mathrm{T}_{\mathrm{F}}=\mathrm{T}_{\mathrm{W}}=240$, $\mathrm{A}=500000, \mathrm{k}_{\mathrm{P}}=500$ und $\mathrm{k}_{\mathrm{I}}=1$ )

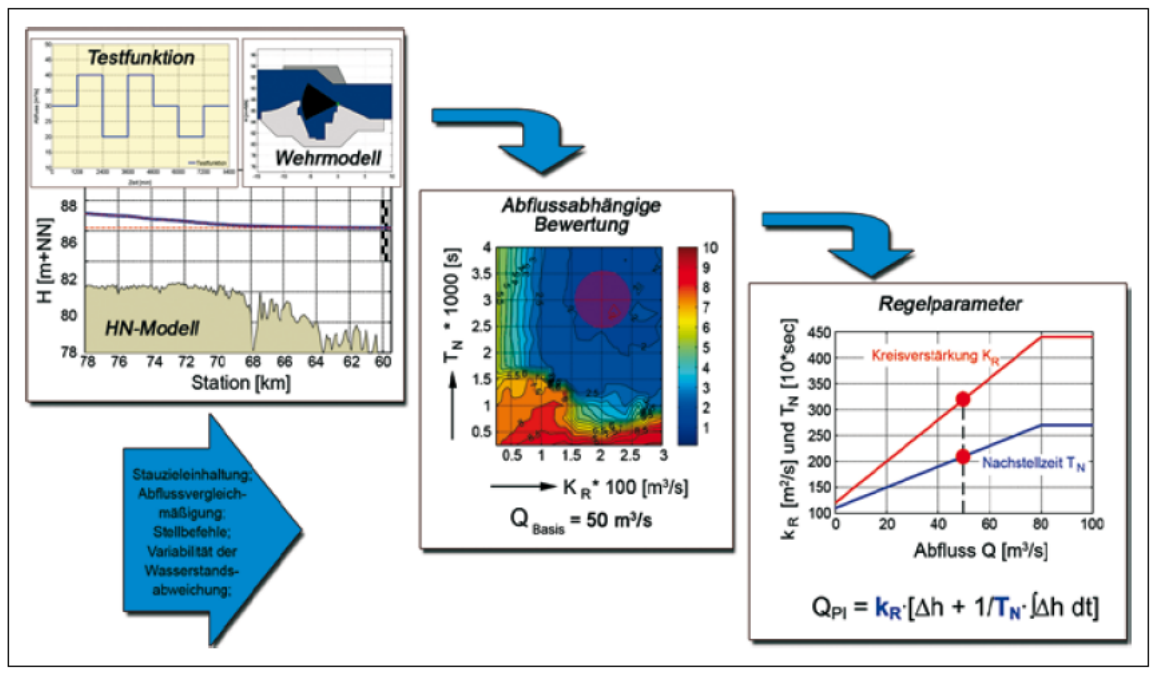

Bild 3: $\quad$ Ablaufdiagramm zur Bestimmung abflussabhängiger Regelungsparameter

Gebhardt, Schmitt-Heiderich: Entwicklung und Simulation von Regelungsalgorithmen für Staustufen an Bundeswasserstraßen. Wasserwirtschaft 6 (2008), S. 16-18.

daraus resultierenden Änderungen im Wasserstand sind in dem linken Diagramm, die der Abflüsse im rechten Diagramm des Bildes 2 dargestellt. Werden lediglich die Regelstrecke und der Regelalgorithmus untersucht (rote Linie), stellen sich bereits nach 1,5 Stunden nahezu stationäre Verhältnisse ein. Der vermeintlich gut parametrisierte Regelkreis zeigt deutliche Abfluss- und 


\section{Autorenfassung}

Gebhardt, Schmitt-Heiderich: Entwicklung und Simulation von Regelungsalgorithmen für Staustufen an Bundeswasserstraßen, 2008

Wasserstandsschwankungen, wenn zusätzlich entweder das Messwertfilter oder die Wehrträgheit berücksichtigt wird (grüne und schwarze Linien). Da für die Abflussänderung q sowohl das Filter als auch das Regelorgan im Vorwärtszweig der Regelungsschleife liegen und ihre Zeitkonstante identisch ist, fallen die beiden Kurven im rechten Diagramm zusammen.

Mit einer realitätsnahen Betrachtung unter Berücksichtigung aller vier Größen des Regelkreises zeigt sich ein aufschwingendes Verhalten (blaue Linie). Die Regelung wird durch die Trägheit des Wehrs instabil und erfordert geänderte Regelungsparameter!

\section{Parametrisierung der lokalen Regler}

Der analytische Ansatz aus Abschnitt 3 eignet sich gut für eine Vorbemessung. Für den Nachweis der Reglerparameter sind allerdings weitere Untersuchungen im mathematischen Modell erforderlich, wie dies in Abschnitt 2 beschrieben wird [4]. Mit dessen Hilfe werden die Parameter abflussabhängig abgeleitet, wobei die Reaktion des Gesamtsystems auf Zuflusstestfunktionen um einen Basisabfluss analysiert wird. Es reicht nicht aus, die Wasserstandsabweichungen gegenüber einem Sollwasserstand zu analysieren. Die Gesamtbewertung berücksichtigtzusätzliche Kriterien, wie die Abflussvergleichmäßigung, Variabilität der Wasserstandsabweichungen, Anzahl der Stellbefehle der Regelungsorgane etc. Alle Kriterien werden auf eine einheitliche Skala normiert und gewichtet zu einer Gesamtbewertung zusammengefasst. Durch Variation der Parameter (hier: $K_{R}$ und $T_{N}$ ) kann der Parameterraum bezüglich der Eignung bewertet werden. In Bild 3 ist eine derartige Gesamtbewertung veranschaulicht. Blaue Bereiche sind geeignet, während rote Bereiche für die Regelung nicht geeignet sind. Die gleiche Prozedur wird auf unterschiedlichen Abflussniveaus wiederholt, um die Regelungsparameter abflussabhängig optimal zu erhalten. Anhand historischer Ereignisse müssen die Parametersätze abschließend den Nachweis erbringen, den Wasserstand entsprechend den Vorgaben zu führen.

Michael Gebhardt and Peter Schmitt-Heiderich

\section{Development and Simulation of Controller Algorithms for Barrages at Federal Water- ways}

The automatic flow and water level control of reservoirs is due to the non-linear behavior of the process and the multi-purpose use of the reservoir a sophisticated control task. In this paper, the methods are presented, which are used in the development and simulation of controller algorithms for barrages at the federal waterways. Moreover it is shown, that modeling the weir for the control design can not be neglected. 


\section{Autorenfassung}

Gebhardt, Schmitt-Heiderich: Entwicklung und Simulation von Regelungsalgorithmen für Staustufen an Bundeswasserstraßen, 2008

\section{Schlussfolgerung und Ausblick}

Im Rahmen der Automatisierung von Staustufen an Bundeswasserstraßen werden in der BAW Methoden und Werkzeuge verwendet, welche die Entwicklung, die systematische Parameteroptimierung und den Test von Regelungsalgorithmen erlauben. Negative Auswirkungen für die Schifffahrt können damit im Vorfeld vermieden und eine Aufrechterhaltung des Verkehrs gewährleistet werden. Nach der Inbetriebnahme ist es wichtig, den automatisierten Betrieb zu beobachten, z. B. durch einen Remote- Zugriff aufdie Anlage: Zum einen zur Erfolgskontrolle und Qualitätssicherung und zum anderen zur Verifikation der Modelle sowie ggf. Korrektur der gewählten Parameter bei abweichenden Verhalten in Modell und Natur. Zur Erhöhung der Akzeptanz beim Betriebspersonal sind Schulungen von Vorteil, in denen besondere Betriebssituationen unter Verwendung von Hardware-in-the-Loop- Simulationen in Echtzeit vorgeführt werden können.

Perspektivisch werden Software-Werkzeuge mehr und mehr an Bedeutung gewinnen, die eine automatische Umsetzung von Steuerungen und Regelungen auf der Hardware-Seite ermöglichen. Bei diesem als Model-Based-Design bezeichneten Entwicklungsprozess wird aus dem Simulationssystem heraus ein ausführbares Regler- oder Steuerprogramm auf der industriellen Zielhardware erzeugt. Eine aufwändige und fehleranfällige manuelle Portierung wird so vermieden. 


\section{Autorenfassung}

Gebhardt, Schmitt-Heiderich: Entwicklung und Simulation von Regelungsalgorithmen für Staustufen an Bundeswasserstraßen, 2008

\section{Formelzeichen:}

$\begin{array}{cc}\mathrm{A} & {\left[\mathrm{m}^{2}\right]} \\ \mathrm{H} & {[\mathrm{m}]} \\ \mathrm{F} & {\left[\mathrm{m}^{3} / \mathrm{s}\right]} \\ \mathrm{Q} & {\left[\mathrm{m}^{3} / \mathrm{s}\right]} \\ & \\ \mathrm{K}_{\mathrm{p}} & {\left[\mathrm{m}^{2} / \mathrm{s}\right]} \\ \mathrm{K}_{\mathrm{I}} & {\left[\mathrm{m}^{2} / \mathrm{s}^{2}\right]} \\ \mathrm{K}_{\mathrm{R}} & {\left[\mathrm{m}^{2} / \mathrm{s}\right]}\end{array}$

Speicheroberfläche

Änderung der Wassertiefe

Änderung des Zuflusses in die Stauhaltung

Änderung des Abflusses aus der Stauhaltung

Regelparameter Proportionalanteil

Regelparameter Integralanteil

Regelparameter Kreisverstärkung

$$
\left(\mathrm{k}_{\mathrm{R}}=\mathrm{k}_{\mathrm{P}}\right)
$$

$\mathrm{T}_{\mathrm{N}}$

[s]

Regelparameter Nachstellzeit

$$
\left(\mathrm{T}_{\mathrm{N}}=\mathrm{k}_{\mathrm{P}} / \mathrm{k}_{\mathrm{I}}\right)
$$

$\mathrm{T}_{\mathrm{F}} \quad[\mathrm{s}]$

Zeitkonstante des Filters

$\mathrm{T}_{\mathrm{W}}$

[s]

Zeitkonstante des Wehres

\section{Autoren}

Dr.-Ing. Michael Gebhardt

Dr.-Ing. Peter Schmitt-Heiderich

Bundesanstalt für Wasserbau

Kussmaulstr. 17

76187 Karlsruhe

michael.gebhardt@baw.de

peter.schmitt-heiderich@baw.de

\section{Literatur}

[1] Grimm, E. et al.: Entwicklungsstand der physikalischen Modelluntersuchung von Schleusen und Wehren. In: Mitteilungsblatt der Bundesanstalt für Wasserbau, Wasserbauliches Versuchswesen, 2007, Heft Nr. 90, S. 9-24. 


\section{Autorenfassung}

Gebhardt, Schmitt-Heiderich: Entwicklung und Simulation von Regelungsalgorithmen für Staustufen an Bundeswasserstraßen, 2008

[2] Kühne, A.: Flussstauregulierung, Grundsätzliche Betrachtungen mit systemtheoretischen Methoden. In: Mitteilungen der Versuchsanstalt f. Wasserbau, Hydrologie und Glaziologie, Technische Hochschule Zürich, 1975.

[3] Kühne, A.: Stauregulierung an Flüssen. In: Wasser- und Energiewirtschaft Nr. 69 (4), 1975.

[4] Bundesanstalt für Wasserbau (Hrsg.): Simulation von Hydraulik und Leittechnik im numerischen Staustufenmodell zur Optimierungder automatisierten Steuerung und Regelung von Wehren und Wasserkraftwerken. In: Tätigkeitsbericht der BAW, 2004, S. 54-58. 